Fourth International Symposium on Physics of Fluids (ISPF4)

International Journal of Modern Physics: Conference Series

Vol. 19 (2012) 73-82

(C) World Scientific Publishing Company

DOI: $10.1142 /$ S2010194512008604

\title{
SHOCK INDUCED SEPARATING FLOWS IN SCRAMJET INTAKES
}

\author{
YUFENG YAO AND DANIEL RINCON \\ School of Aerospace and Aircraft Engineering, Kingston University \\ Roehampton Vale, Friars Avenue London SW15 3DW, UK \\ y.yao@kingston.ac.uk \\ YAO ZHENG \\ School of Aeronautics and Astronautics, Zhejiang University \\ Hangzhou Zhejiang, 310027, P.R. China
}

\begin{abstract}
Shock induced separating flows in a scramjet intake has been studied by using a computational fluid dynamics approach. A configuration of scramjet intake geometry consisting of two exterior compression ramps, followed by a subsequent inlet and interior isolator/diffuser assembly, is chosen. The flow conditions are incoming Mach 7 with free-stream static temperature of $46.3 \mathrm{~K}$ and wall temperature of $300 \mathrm{~K}$, respectively. Consequently, oblique shock wave will be formed and its interaction with viscous boundary layers will lead to flow separation that is responsible for the loss of mass flow, total pressure and several other effects. Simulations confirmed that it is necessary to include the cowl wedge in order to predict wall pressure distributions along the lower surface of the intake walls. It is also shown that mesh topology has some influences on prediction results with structured mesh gives better predictions than that of hybrid mesh. A total of eight turbulence models have been applied and results have shown reasonably good agreement with the experimental measurements and other numerical predictions, with small differences occur in localized regions particularly after shock reflection inside the intake channel. Not surprisingly, shock induced flow separation occurs and it correlates well with higher wall pressure and heating downstream. This phenomenon will have significant impact on deteriorating boundary layer property and consequently the engine performance.
\end{abstract}

Keywords: Scramjet; supersonic flow separation; shock-wave/boundary-layer interactions.

\section{Introduction}

A supersonic combustion ramjet (scramjet) is a variant of a ramjet air-breathing combustion jet engine. In scramjet, the heat addition, due to the combustion process, occurs in an airflow that is supersonic relative to the engine. As to ramjets, conceptually, a scramjet relies on a higher vehicle flying speed to forcefully compress the incoming airflow and then decelerate it before combustion. Whereas a ramjet decelerates airflow to 'subsonic' velocities, the airflow in a scramjet still remains 'supersonic' throughout the entire engine cycle. This enables the scramjet to operate at extremely high-speeds more efficiently. For example, using a hydrogen fuel and variable geometry, theoretical projections estimate the top speed of a scramjet-powered hypersonic vehicle between 
Mach 12 and Mach 24, which is near the orbital velocity (Smart, 2008). Figure 1 illustrates a traditional scramjet engine type, in which an incoming airflow at supersonic or hypersonic speeds is diffused through an intake channel to a lower, but still supersonic speed at station 4, where fuel (either liquid or gas) is injected from the walls (via holes, slots, pylons) and/or in-stream protuberances (via struts, tubes, pylons), then it mixes with supersonic airflow and burns in a generally diverging area of combustor between station 4 and station 5, before finally exits into atmosphere after the nozzle outlet.

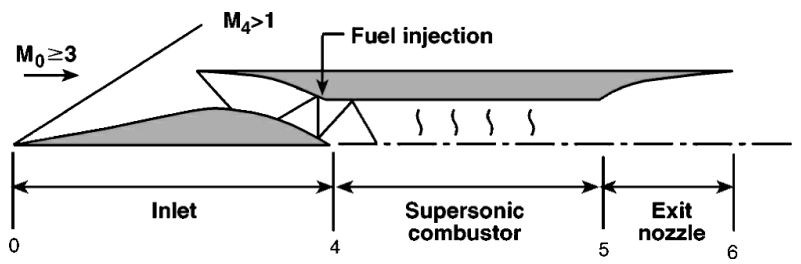

Fig. 1. Schematic view of a generic supersonic combustion engine.

Of many research areas in scramjet flow study, particular interest has been placed on to the evaluation of aerodynamic performance of the boundary-layer transition on the fore-body and complex shock-wave/boundary-layer interactions (SBLI) in the intake, both problems are crucial for understanding and modeling supersonic combustion of resultant high engine thrust. The present study will focus on the latter issue.

The intake of a scramjet often consists of several exterior compression ramps, followed by a subsequent inlet and interior isolator/diffuser assembly. A series of oblique shock waves will be formed that compress the incoming airflow. The interaction of these strong shock waves with thickened hypersonic boundary layers will cause larger flow separation zones that are responsible for considerable loss of mass flow, total pressure and several other effects, such as unsteady shock-wave movement and higher surface heat flux into the structural materials of the engine. Meanwhile, high total enthalpy of the airflow will also yield severe aerodynamic heating, being further enhanced by turbulent heat flux (Krause, Reinartz and Ballmann, 2006). All these flow phenomena can deteriorate boundary layer property and cause significant flow separation. Hence, the characteristics of scramjet intake flows has critical responsible for the whole engine performance and this is the reason why the modeling and simulation of SBLI intake flow is of great importance in scramjet researches.

Several candidates for scramjet intake flow study were discussed in a review paper by Scheider (2004). It was suggested that one case would be axisymmetric geometries such as that of the Russian Kholod vehicle (Ito et al. 2001) and another likely candidate would be geometries similar to the NASA Hyper-X (Berry et al. 2000, 2001). In particular, it was found from a study of the Hyper-X model that upstream of the first corner, the boundary-layer flow is nearly streamwise, with little spanwise cross-flow movement. The laminar boundary layer entering the first corner forms a separation bubble. The bubble affects the flow near the surface, as does the change in spanwise pressure gradient presumably associated with the increased wedge angle downstream of the corner. The 
cross-flow is induced in the boundary layer downstream of the first corner, with the amount of cross-flow that occurs being dependent on the properties of the boundary layer entering the first ramp, downstream of the first corner. The second corner does not seem to cause any dramatic changes. Since the Mach 6 Oil-flow seems generally similar to the Mach 10 data, these appear to be generic effects.

There are numerous computational studies of scramjet intake flow at incoming flow Mach number ranging from 2.5 to 10 with specific interest on Mach 7 flow. Reinartz et al. (2006) investigated double wedge shock boundary layer interactions by using a combined experimental as well as computational analysis of hypersonic flows over heated ramp and wedge configurations. The numerical simulation used two wellvalidated Reynolds averaged Navier-Stokes solvers with a variety of turbulence models. Different surface temperatures are specified to investigate the impact on the shock-wave/ boundary-layer interaction and on the size of the separation. To analyze the effect of an entropy layer behind a blunt leading edge on the structure of the boundary layer as well as on the development of the inviscid flow field, flows over double wedge configurations with different nose radii are computed and compared to the experimental results. There are two test configurations being investigated which both have been designed for and tested in shock tunnel experiments. The first one is a heated compression ramp model with a ramp angle of $15^{\circ}$. Due to the heating of the model, the wall temperature can be explicitly prescribed and varied over different test runs to study in detail the influence of the wall temperature on the flow field. The second configuration is a heated double wedge with different nose radii. Figure 2 shows a schematic of the model and the flow characteristics. Increased blunting of the leading edge of the first wedge during the test runs shows the effect of a growing entropy layer. The conical nozzle of the shock tunnel produces an expanding flow with specific gradients for all flow variables given. The test conditions at the model inlet correspond to $M_{\infty}=7.42$ and $T_{0}=2720 \mathrm{~K}$ as well as $M_{\infty}=8.3$ and $T_{0}=1420 \mathrm{~K}$ in the test section, respectively. The experimental set-ups were designed to yield nominally two-dimensional laminar flow. Comparison with the thereby obtained experimental surface distributions and Schlieren pictures allows the validation of the numerical work. So far, only quasi steady and two-dimensional as well as preliminary $3 \mathrm{D}$ computations have been performed. However, further analysis is currently under way investigating the effect of spillage and unsteadiness in the flow by running threedimensional and time-accurate computations. Subsequently, the computations can be used to investigate parameter variations that are not possible within experiments, e.g. the variation of $R e_{\infty}$ (i.e., scaling of the model) and simultaneously keeping $M_{\infty}$ and $T_{W} / T_{0}$ constant, and to develop trends. Test results include $S_{t}$ and $C_{p}$ distributions, etc. Furthermore, the influence of round leading-edges and flow transition on the intake flow has been studied by Krause, Reinartz and Ballmann (2006) and Krause and Ballmann (2008), respectively. 


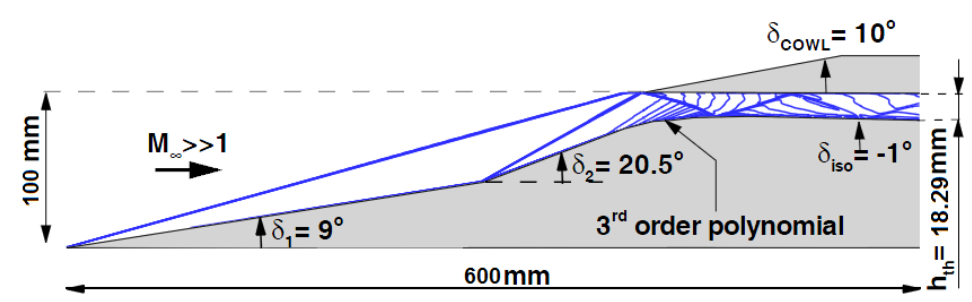

Fig. 2. Hypersonic intake flow model with sharp double wedge configuration.

In this work, a computational fluid dynamics (CFD) study of scramjet intake flow will be carried out on a representative model proposed by Reinartz et al. (2006) (Fig. 2). Simulations will be performed to demonstrate influence of mesh topology, grid convergence, and turbulence model effect. The CFD predicted wall properties will be compared with available test data and the shock-induced separated flow structure will be revealed as well.

\section{Problem Description and Numerical Approach}

The configuration being investigated was designed and has been tested in shock tunnel experiment by Reinartz et al. (2006) as seen in Fig. 2. It comprises a double wedge compression ramp with the first compression ramp of 9 degree wedge angle and the second one of 20.5 degree, respectively. This is followed by a curved wedge shoulder with the $3^{\text {rd }}$ order polynomial fitting and then ends by a long channel at a small expansion angle of 1 degree. The cowl wedge has an angle of 10 degree with its lower wall surface kept horizontal. The model has a total length of model of $600 \mathrm{~mm}$, inlet height of $100 \mathrm{~mm}$, and exit throat of $18.29 \mathrm{~mm}$. The oblique shock-wave due to the first ramp will be formed and impinge on to a location close to the leading-edge of the cowl wedge. The second oblique will also be formed at the corner of the compression ramp and impinges to a location in the vicinity of the cowl leading-edge. Due to the cowl wedge, shock-wave deflection occurs and reflected inside the channel, forming a so called shock-train. Around the expansion corner, flow separation will occur due to shock-wave/boundarylayer interactions and this will lead to higher wall surface heat flux, in some extreme conditions, will cause serious turbulent heating. The flow conditions are incoming freestream flow of Mach 7, density of $0.0127 \mathrm{~kg} / \mathrm{m}^{3}$, static temperature of $46.3 \mathrm{~K}$; this results in a unit Reynolds number of $4.0 \mathrm{E}+6$. The wall temperature of $300 \mathrm{~K}$ is kept as constant in all simulations (Krause and Ballmann, 2008).

Numerical approach applies the Reynolds-averaged Navier-Stokes (RANS) governing equations with turbulence model as a closure. Due to moderate shock-viscous interaction, unsteadiness of shock-wave and separated flow is assumed to be small; thus simulation considers steady RANS with emphasis being primarily placed on grid refinement study, mesh topology influence, turbulence model effect on the prediction results. Hybrid mesh (not shown) was generated using ANSYS CFX-meshing tool with structured grid in the near wall region and unstructured grid away from wall (ANSYS, 2009). Later, three set of structured grids were generated using ANSYS Gambit meshing 
tool. Figure 3 illustrates a baseline structured mesh of about 182,000 elements with two further grid refinement in the streamwise and the wall-normal directions, respectively. All simulation uses high-resolution $\left(2^{\text {nd }}\right.$ order) scheme and runs in double precision. A residual of $10^{-5}$ was set and in general this convergence criterion can be achieved within $\max 200$ iterations.

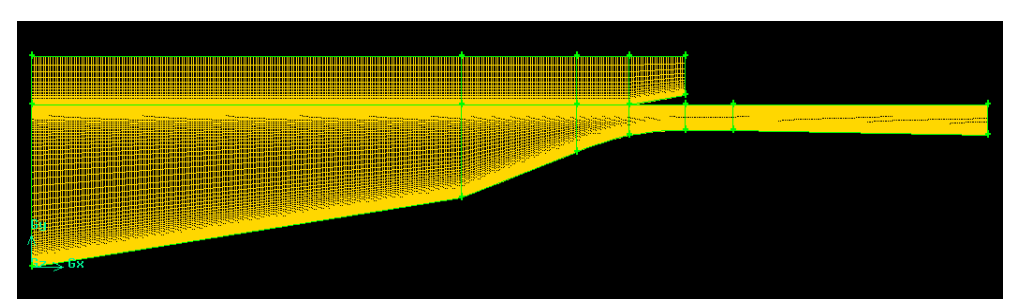

Fig. 3. Structured mesh generated by ANSYS Gambit.

\section{Results and Discussions}

The CFD predicted results will be compared with experimental measurement and other available numerical predictions for the same configuration. The focus will be placed on wall properties and separated flow structures.

\subsection{Mesh topology and domain influence studies}

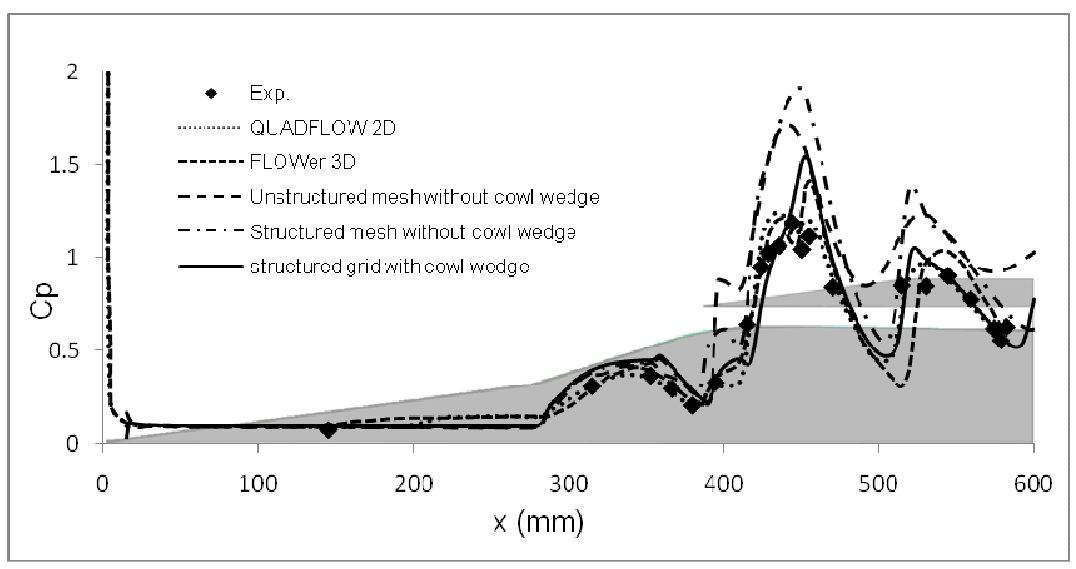

Fig. 4. Influence of mesh topology and domain in comparison to experimental data and other CFD results.

Figure 4 illustrates CFD predicted wall pressure coefficient distributions from hybrid mesh of a domain without cowl wedge (denoted as mesh 2), structured mesh of a domain without cowl wedge (denoted as gambit mesh) and structured mesh of a domain with cowl wedge (denoted as gambit mesh + new domain). It appears that for a small domain without cowl wedge, higher pressure jump was captured by both hybrid and structured meshes and this is due to strong reflected shock-wave whilst it impinges onto the upper surface of the domain. While a full domain including cowl wedge was applied, oblique 
shock waves formed from the first and the second ramps will pass through this horizontal plane and end at the exit plane above the cowl wedge without reflecting back to the flow domain. This results in a more realistic wall pressure distribution as shown in Fig. 4, in consistent with other numerical results. Hence, a full computational domain with cowl wedge included will be used for the remaining simulations.

\subsection{Grid convergence study}

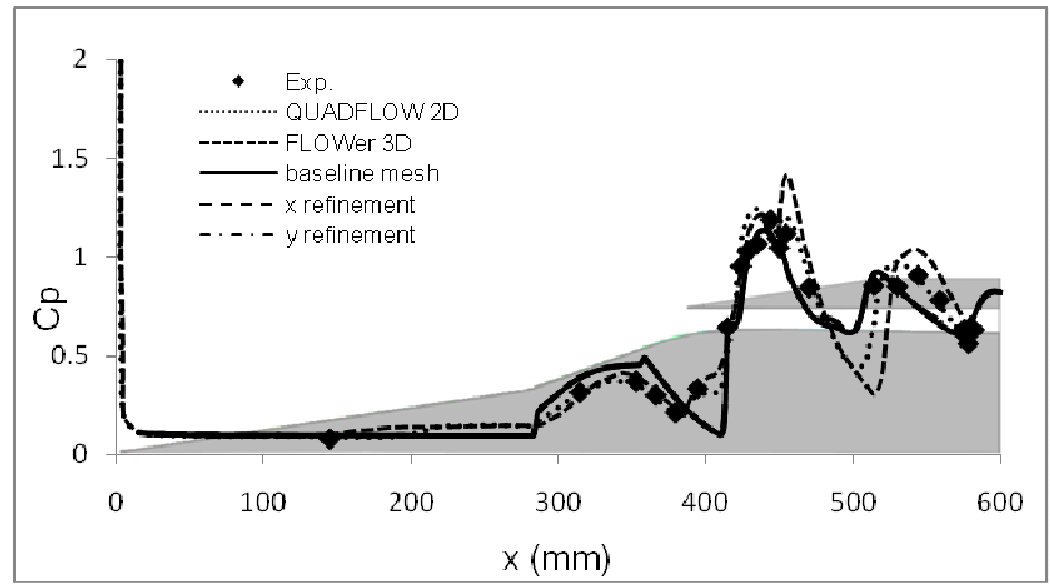

Fig. 5. Comparison of grid refinement study of baseline mesh, x-refinement and y-refinement meshes.

The baseline structured mesh consists of 182,000 elements in ten blocks as seen in Fig. 3. Further two grids were generated by refining grids in the streamwise (x) and the wallnormal (y) directions, respectively, by appropriately $50 \%$ extra grid points. This results in about 270,000 elements for the x-refinement and the y-refinement grids. Comparisons of the wall pressure distribution (Fig. 5) have shown good agreement with very small differences around $x=490,590 \mathrm{~mm}$. It is thus concluded that the baseline mesh is fine enough for this case study and it will be used for turbulence model assessment.

\subsection{Turbulence model assessment}

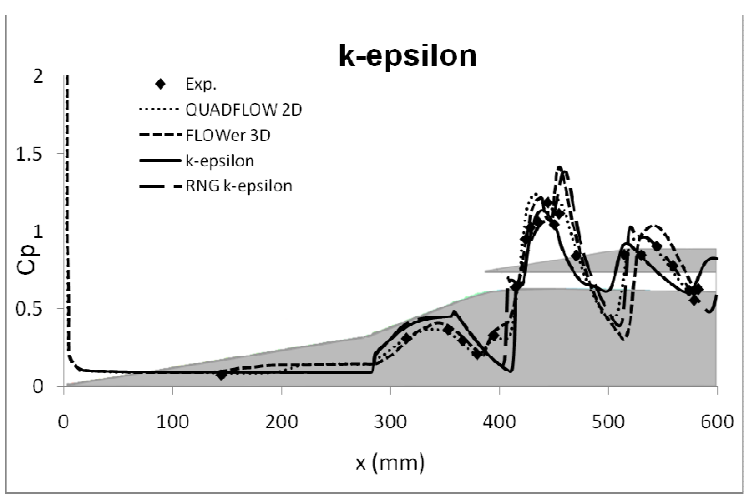



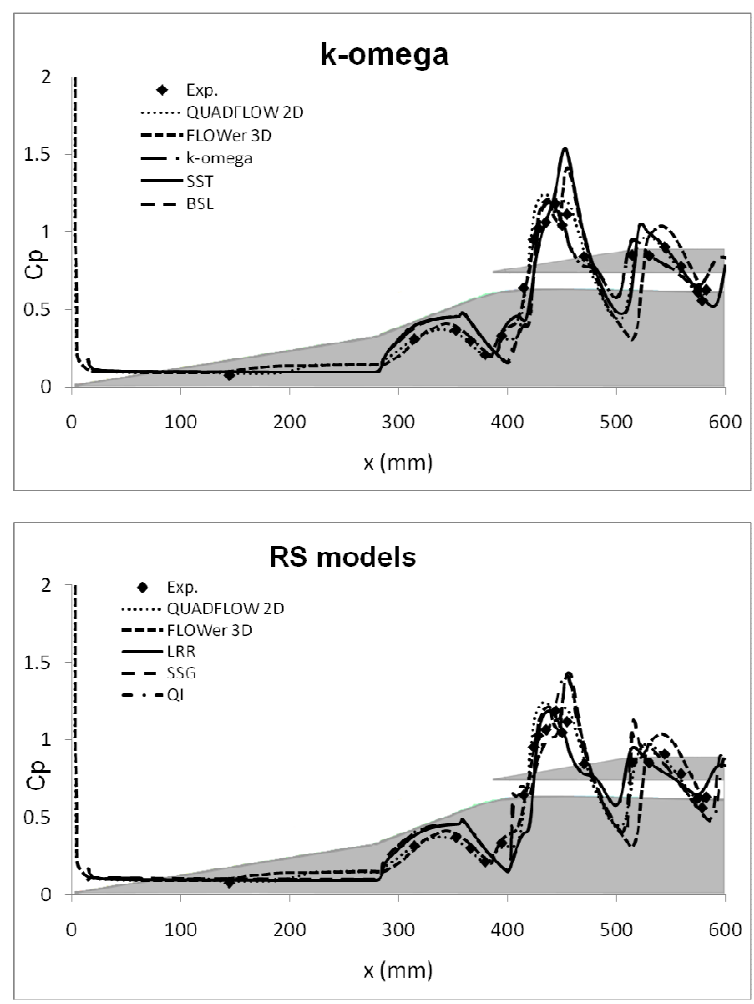

Fig. 6. Influence of turbulence model on wall pressure distributions.

A total of eight turbulence models have been adopted for model influence assessment. Based on the property of these models, the comparisons were made in three groups; i.e. $\mathrm{k}-\varepsilon$ model family (standard k- $\varepsilon$ model, re-normalized group RNG k- $\varepsilon$ model), k- $\omega$ model family (standard k- $\omega$ model, shear-stress turbulence SST model, and baseline BSL k- $\omega$ model), and the Reynolds stress model family (SSG, LRR, QI models) (ANSYS, 2009). Results from these three types of turbulence models are broadly in reasonable good agreement with test data and other numerical predictions, and differences are mainly in localized areas inside the intake channel, in terms of shock impingement location, impinging shock and reflecting shock strengths, etc. Overall, the RNG k- $\varepsilon$ model, the SST model and the SSR/QI model predictions are in better agreement with other numerical results, while the $\mathrm{k}-\varepsilon$ model, the $\mathrm{k}-\omega$ model, the BSL $\mathrm{k}-\omega$ model, and the LRR model are in better agreement with the experimental measurements.

\subsection{Wall skin friction and heat transfer}

Figure 7 (left) gives comparison of skin friction coefficient distributions by three turbulence models two-equation type RNG k- $\varepsilon$ and SST models and Reynolds stress LRR model. Both RNG k- $\varepsilon$ and LRR models capture small separation bubble after reflection shocks around $\mathrm{x}=400 \mathrm{~mm}$, while SST model predicts larger bubble with separation point 
further upstream and bubble length about 2-3 times compared to that of LRR prediction. Furthermore, an incipient flow separation has been captured by SST model around the corner of ramp $x=280 \mathrm{~mm}$. Figure 7 (right) shows the Stanton number distributions from standard k- $\varepsilon$ and RNG k- $\varepsilon$ models and Reynolds stress models of LRR and SSG. It can be seen that in the regime of flow separation, Stanton number shows large amplitude of oscillation, and downstream it follows similar pattern as that seen in pressure coefficient distributions, in consistent with impinging and reflecting shock waves inside the channel.
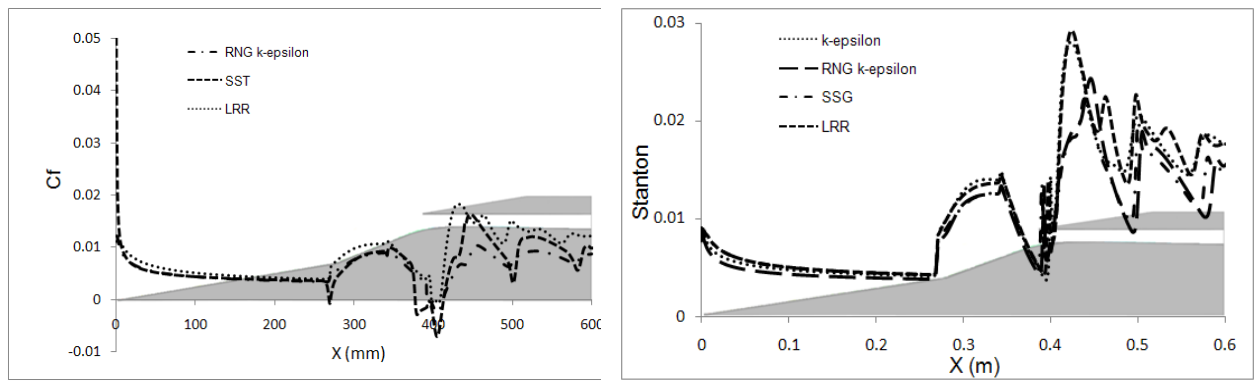

Fig. 7. Comparison of skin friction coefficient and Stanton number distributions.

\subsection{Flow structures in separating region}

The scramjet intake flow is featured by the formation of oblique shock-wave from both the leading-edge of the first ramp and also the compression corner between the first and the second ramps, and shock impingement on to developing boundary-layer as shown in Fig. 8 (left). A separation bubble is formed due to strong interactions of shock-wave and viscous layer. A close view of velocity vector clearly shows the bubble boundary in terms of streamlines with reverse flow in near wall region (Fig. 8 (right)). It is also found that the flow separation $\left(\mathrm{x}_{\mathrm{s}}\right)$, center of the bubble $\left(\mathrm{x}_{\mathrm{c}}\right)$, and flow reattachment $\left(\mathrm{x}_{\mathrm{r}}\right)$ locations are slightly influenced by the turbulence model as shown in Fig. 9. For four models shown (SST, RNG k- $\varepsilon$, LRR, and BSL- $\gamma$ ), $\left(\mathrm{x}_{\mathrm{s}}, \mathrm{x}_{\mathrm{c}}, \mathrm{x}_{\mathrm{r}}\right)=(376.6$, 401.2, 412.9), (399.7, 405.9, 411.4), (397.8, 405.6, 410.0), (384.7, 403.8, 411.4), resulting a bubble length of $36.3 \mathrm{~mm}$, $11.7 \mathrm{~mm}, 12.2 \mathrm{~mm}$, and $26.7 \mathrm{~mm}$, respectively. There is roughly a factor of three in terms of predicted bubble length between different models. The SST model predicts largest bubble height of $6.28 \mathrm{~mm}$, representing about $60 \%$ throat height and $40 \%$ blockage.
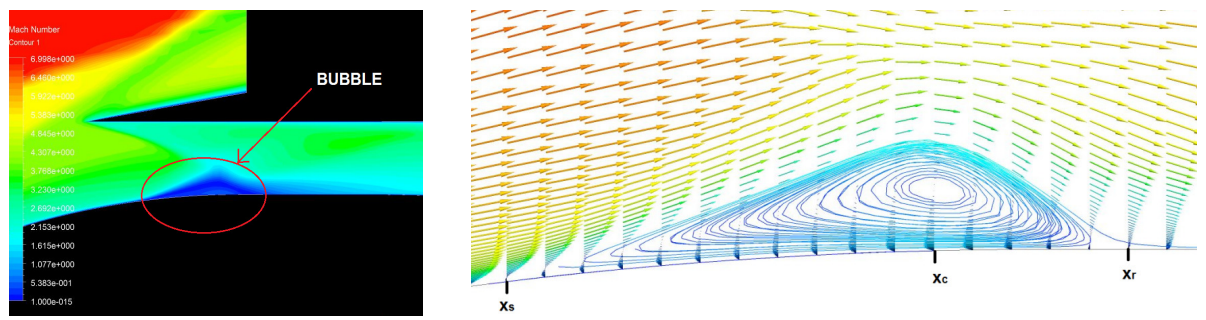

Fig. 8. Contours of Mach number (left) and velocity vectors superimposed with streamlines (right) illustrating shock-induced separation bubble from simulation using standard k- $\varepsilon$ model. 


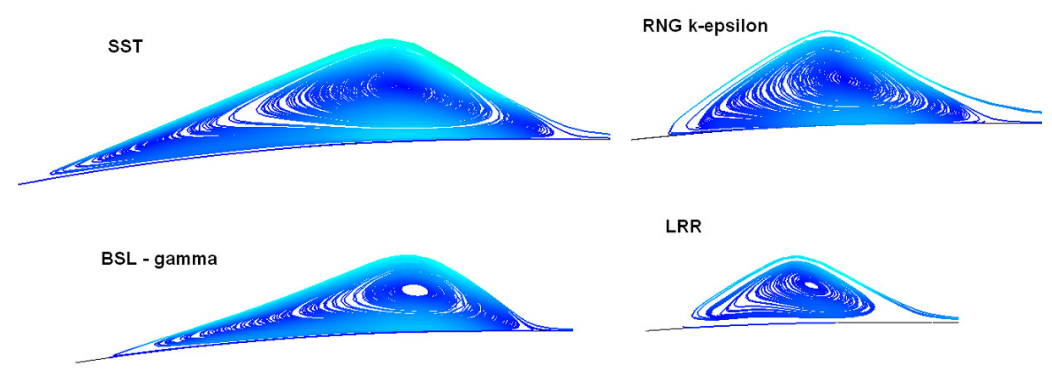

Fig. 9. Size and shape of separation bubble produced by four different turbulence models.

\subsection{Effect of leading-edge radius}

Influence of rounded leading edges on the intake flow has been studied with radius of 0.5 for the leading edge of the first ramp and the cowl wedge. It is expected that detached bow shock waves will be formed in front of the first ramp and the cowl wedge. Similar to that of sharp leading edges, the bow shocks will impinge on to the boundary layer inside the intake channel and induce flow separation. Figure 10 shows comparisons of simulated wall pressure and skin friction coefficients distributions using SST turbulence model. Broadly, results are quite similar except that in the case of rounded leading edges earlier pressure recovery occurs with larger separation bubble captured. Results from other turbulence model have shown similar trends (not shown here).
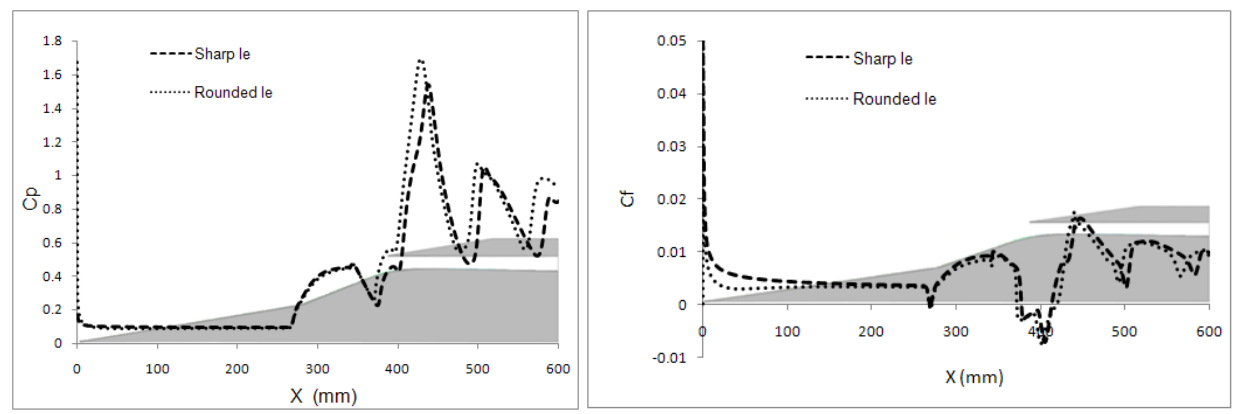

Fig. 10. Comparison of wall pressure and skin friction coefficient from sharp and round leading-edge.

\section{Conclusions}

Shock induced separated flow inside a scramjet intake configuration has been studied by using a computational fluid dynamics approach of solving the Reynolds-averaged NavierStokes equations. At Mach 7 incoming flow and unit Reynolds number of 4.0E+6, two different intake geometries have been studied; i.e. sharp and rounded leading edges. It was found that difference caused by the turbulence models were greater than that due to the change of the leading edges. Furthermore, turbulence model has yielded quite similar wall pressure coefficient distribution, but it has significant impact on flow separation 
bubble size and its extent with a factor of three in difference between different models. Large flow separation will lead to significant blockage of intake flow, thus it is necessary to modify the expansion shoulder geometry in order to reduce large flow separation.

\section{Acknowledgments}

The third author would like to acknowledge the sponsorship provided by the ERASMUS program of the European Union.

\section{References}

1. M. Smart, RTO-EN-AVT-150, pp. 9.1 - 9.38 (2008).

2. M. Krause, B. U. Reinartz and J. Ballmann, AIAA Paper, 2006-8137 (2006).

3. S. Schneider, Prog. Aero. Sci. 40, 1 (2004).

4. T. Ito, L. A. Randall, S. P. Schneider, J. Spacecraft Rockets, 38, 692 (2001).

5. S. Berry, A. Auslender, A. D. Dilley and J. Calleja, AIAA Paper, 2000-4012 (2000).

6. S. Berry, A. Auslender, A. D. Dilley and J. Calleja, J. Spacecraft Rockets, 38, 853 (2001).

7. B. U. Reinartz, J. Ballmann and R. Boyce, AIAA Paper, 2006-8137 (2006).

8. M. Krause, B. U. Reinartz and J. Ballmann, $25^{\text {th }}$ Int. Cong. Aeronau. Sci. ICAS-5.2.3, Hamburg, Germany (2006).

9. M. Krause, M. Behr and J. Ballmann, AIAA Paper, 2008-2598 (2008).

10. ANSYS v12 manual, ANSYS Ltd. (2009). 